\title{
Frequência de dor lombar em operadoras de telemarketing em Santa Maria, RS
}

\author{
Hedioneia Maria Foletto Pivetta ${ }^{1}$, Ana Paula Ziegler Vey²
}

\begin{abstract}
RESUMO
O objetivo do estudo foi avaliar a frequência de dor lombar em operadoras de telemarketing de uma empresa da cidade de Santa Maria/RS, bem como sua situação laboral e capacidade funcional das funcionarias que possuírem dor lombar. Os instrumentos utilizados para a coleta de dados foram: a ficha de avaliação além dos questionários de Avaliação Funcional de Oswestry e de Roland-Morris. Fizeram parte da amostra oito funcionárias, com idade média de 25 anos. Das oito voluntárias, cinco apresentaram média de dor lombar $5( \pm 2,44)$ na escala visual analógica; no questionário Roland Morris a pontuação média foi de $2,8( \pm 2,48)$ e no questionário de Oswestry de $7 \%$ de incapacidade. Pode-se concluir que a dor lombar esteve presente em cinco das oito funcionarias avaliadas, caracterizando-se como dor de média intensidade. A situação laboral identificada permite inferir que as operadoras de telemarketing trabalham na posição sentada e possuem pouco tempo de trabalho na empresa. O maior cansaço identificado foi o mental. 0 índice de incapacidade funcional baixo.
\end{abstract}

Descritores: Dor Lombar; Fisioterapia; Saúde do Trabalhador

\section{Frequency of low back pain in operators telemarketing in Santa Maria, RS}

\begin{abstract}
The aim of the study was to evaluate the frequency of back pain in telemarketing operators of a company from Santa Maria / RS, as well as their employment status and functional capacity of employees who possess low back pain. The instruments used for data collection were: the evaluation form questionnaires beyond the Functional Assessment of Oswestry and Roland-Morris. The sample consisted of eight employees, with an average age of 25 years. Of the eight volunteers, five had average back pain $5( \pm 2,44)$ on the visual analogue scale; Roland Morris questionnaire in the average score was $2.8( \pm 2,48)$ and the Oswestry questionnaire $7 \%$ disability. It can be concluded that low back pain was present in five of the eight evaluated employees, characterized as average pain intensity. The identified employment situation can be inferred that the telemarketing operators work in a seated position and have little time working in the company. The largest strain identified was mental. The low rate of functional disability.
\end{abstract}

Descriptors: Low Back Pain; Physiotherapy; Occupational Health. 


\section{Introdução}

A inserção da tecnologia no ambiente de trabalho aumentou a sobrecarga laboral, sendo que os funcionários necessitam manter posturas sentadas por um período maior de tempo ${ }^{1,2}$. Esse trabalho especializado impõe ao corpo posturas paradoxais em que segmentos como a coluna vertebral permanecem estáticos por longos períodos enquanto os membros superiores precisam realizar movimentos altamente elaborados e repetitivos ${ }^{3,4}$.

Além das mudanças no modo de execução das atividades laborais, a mulher, em especial, conquistou seu espaço no mercado de trabalho. Segundo dados da Fundação Carlos Chagas ${ }^{5}$, em 1970 apenas 18\% das mulheres brasileiras trabalhavam, já em 2002 a metade dessas mulheres estavam inseridas no mundo do trabalho. Exposta as variações hormonais mensais, as mulheres possuem constituição corporal distinta da dos homens. Somado a isso, o sedentarismo e a manutenção da postura predispõe a quadros álgicos. Assim, a mulher do século XXI, que trabalha em setores administrativos pode estar exposta a lesões ocupacionais que podem levar as dores nas costas.

É esperado que $80 \%$ da população mundial sofrerá de dor nas costas, em alguma fase da vida, geralmente na região lombar, três vezes mais frequentemente do que dor na região superior do dorso ${ }^{6}$. Essa dor possui múltiplos fatores, entre eles a adoção de má postura, sedentarismo, movimentos repetitivos no ambiente de trabalho, nos afazeres domésticos, entre outros fatores associados, incluindo a execução errônea de exercícios ${ }^{7,8}$.

A partir do delineamento dos múltiplos agentes danosos no ambiente de trabalho, pressupõe-se condicionante o olhar de pesquisadores da área de saúde voltado ao trabalhador, buscando, através de seus estudos, melhoria nas condições de trabalho e de vida destas pessoas. No contexto da área da saúde, destaca-se a fisioterapia, profissão que possui raízes na reabilitação física e que vem ganhando espaço no ambiente do trabalho, especialidade surgida diante da necessidade do acompanhamento da saúde do trabalhador em geral ${ }^{9}$.

Dentre os serviços em que mais mulheres estão inseridas e a atividade laboral se dá quase que em sua totalidade na posição sentada, podem-se destacar os serviços de teleatendimento, que ganhou impulso no Brasil no final dos anos 1980 e, progressivamente, vem sendo incorporado a vários setores da economia. É uma nova atividade, nascida da necessidade de se ganhar tempo e de se reduzir distâncias ${ }^{10,11}$.

Com relação aos trabalhadores que exercem atividade de teleatendimento, o Código Brasileiro de Ocupações (CBO), agrupa e classifica essas ocupações sob o termo "Operadores de telemarketing" com a seguinte descrição de atividade: Atendem usuários, oferecem serviços e produtos, prestam serviços técnicos especializados, realizam pesquisas, fazem serviços de cobranças e cadastramento de clientes, sempre via teleatendimento, seguindo roteiros planejados e controlados para captar, reter ou recuperar clientes ${ }^{12}$. A realidade organizacional nas centrais de teleatividades busca atualmente a maximização dos resultados, porém, consequentemente, vem gerando impactos sobre a saúde dos seus trabalhadores. Sendo assim, o objetivo deste estudo foi avaliar a frequência de dor lombar em operadoras de telemarketing bem como sua capacidade funcional e a situação laboral destas trabalhadoras.

\section{Metodologia}

A pesquisa foi do tipo descritiva exploratória de cunho quantitativo. A população do estudo foi composta por funcionárias operadoras de telemarketing de uma empresa de Call Center localizada uma cidade do interior do RS. Foram considerados como critérios de inclusão: profissionais operadoras de telemarketing, do gênero feminino, com idade entre 20 e 40 anos, que trabalha sem em uma empresa de Santa Maria, com no mínimo seis meses de trabalho no setor e que cumpram uma carga horária de no mínimo 30 horas semanais.

Após contato e autorização da empresa (a única existente na cidade), os questionários foram entregues para o gerente (por solicitação do mesmo), que direcionou para cada funcionária. A referida empresa é constituída por um total de 18 funcionários (entre técnico administrativos e operadores), sendo que destes oito são operadores de telemarketing, sendo que todas são do gênero feminino e concordaram em participar do estudo. Foi agendada uma data para o recolhimento dos instrumentos (Termo de Consentimento Livre e Esclarecido (TCLE) e questionários), de dez dias após a entrega.

- Foram utilizados para coleta de dados:

- $\quad$ Ficha de avaliação adaptada de Queiroga ${ }^{13}$, composta pelos dados de identificação, sócio-demográficos de cada participante, dados sobre as condições de trabalho, situação laboral e dor músculo esquelética (Escala Análogo Visual EVA e diagrama corporal) ${ }^{14}$. 
- Questionário Roland Morris utilizado para avaliar o desempenho funcional das participantes. Este Questionário é composto por 24 alternativas a serem assinaladas com o objetivo de relatar as situações cotidianas e laborais que podem estar comprometidas pela dor lombar. Quanto maior o número de itens assinalados, pior o estado de saúde do avaliado ${ }^{15,16}$.

- Questionário de Avaliação Funcional de Oswestry, sendo uma ferramenta composta por dez sessões com perguntas constituídas de 6 alternativas com escore correspondente que varia de zero a cinco pontos de acordo com a intensidade e gravidade da dor e comprometimento da coluna lombar. 0 trabalhador foi instruído a assinalar, em cada sessão, a alternativa que melhor representasse seu estado físico. 0 resultado final foi obtido em porcentagem por meio da fórmula: soma dos escores das sessões x $100 \div 50$, onde 50 representa o escore máximo possível. Os valores mais altos se referem à maior incapacidade do indivíduo ${ }^{17}$.

Os dados foram analisados por meio da estatística descritiva simples das respostas dos questionários. 0 projeto de pesquisa obteve aprovação do Comitê de Ética e Pesquisa com seres humanos, protocolo CAAE n 12407113.4.0000.5346.

\section{Resultados}

Das oito operadoras de telemarketing, todas responderam a pesquisa e assinaram o TCLE . A idade média foi de 25 $\pm 6,05$ anos, sendo que somente duas tinham filho e duas eram tabagistas.

A análise da ficha de avaliação com relação a situação laboral atual permitiu identificar que a média de trabalho em anos na empresa é de 2,6 \pm 1,9 anos, sendo que todas desenvolvem suas atividades laborais na posição sentada, durante 6 horas diárias com dois intervalos de dez minutos. Ainda quanto à situação laboral, quando questionadas ao cansaço produzido pela função, todas referiram sentir ao final do dia de trabalho e, quanto a natureza do cansaço, seis das mulheres manifestaram cansaço mental, uma somente cansaço físico e uma manifestou ambos (físico e mental).

Já com relação a demanda exigida pela função, cinco considerou a demanda mental, sendo que três consideraram que a demanda de trabalho exigida foi física e mental. Outro dado que a pesquisa evidenciou foi o alto índice de sedentarismo das participantes, sendo que sete delas não realizava atividade física regular.

Quando questionadas acerca da dor músculo esquelética, cinco das mulheres responderam afirmativamente, sendo a média assinalada na EVA de $5 \pm 2,44$, considerada como dor de média intensidade ${ }^{14}$. Quando perguntadas se a dor ocasionava impedimento para frequentar o trabalho, somente duas responderam afirmativamente.

Os resultados do questionário Roland Morris utilizado para avaliar o desempenho funcional, cujos valores variam de zero (totalmente capaz apesar da dor) e 24 (totalmente incapaz por causa da dor nas costas), demonstram escores baixos, inferindo que a dor manifestada pouco interfere na capacidade funcional destas pesquisadas, conforme dados da Tabela 1.

Tabela 1 - Nível de incapacidade funcional de mulheres com dor lombar

\begin{tabular}{c|c}
\hline Roland Morris & $\mathrm{N}$ \\
\hline $0 \cdot-6$ PONTOS & 7 \\
\hline $7 \cdot-13$ PONTOS & 1 \\
\hline $14 \cdot-24$ PONTOS & 0 \\
\hline TOTAL & $\mathbf{8}$ \\
\hline
\end{tabular}

A média do nível de incapacidade foi de 2,8 \pm 2,4 pontos, sendo considerado baixo para o nível de incapacidade funcional.

Com relação ao questionário de Oswestry, a média do escore encontrado na população estudada foi de $7 \pm 6,4 \%$ de incapacidade sendo o mínimo zero e máximo mencionados nos escores 14 , sendo considerada incapacidade mínima apesar da presença de dor lombar.

\section{Discussão}

A amostra estudada apresentou características laborais que se reportam a postura sentada, com carga horária diária de seis horas com dois intervalos de dez minutos. A posição sentada possibilita pouca margem de movimentação, tendo 
como consequência carga estática sobre certos segmentos corporais. A postura sentada, por melhor que seja, impõe uma carga biomecânica sobre os discos intervertebrais, principalmente na região lombar ${ }^{18,19}$.

É sabido que a posição sentada aumenta a pressão intra discal, elevando assim o risco de hérnia no disco intervertebral ${ }^{20}$. As cargas na coluna são sempre maiores na posição sentada do que na postura em pé, devido aos elementos posteriores da coluna vertebral que formam uma carga ativa quando em pé. No entanto, na posição sentada não há participação destes elementos de força anti gravitacional permitindo assim que os discos recebam uma carga maior ${ }^{21 .}$

Trabalhar sentado não é assim tão inofensivo como pode parecer à primeira vista. Os autores Viel e Esnault22, afirmam que no decorrer do tempo o conceito de trabalho e a natureza do mesmo mudaram consideravelmente, pois as atividades em pé foram substituídas gradualmente pelas atividades sentadas, e consequentemente os indivíduos que permanecem longos tempos sentados como escolares, funcionários técnico administrativo, motoristas e todos aqueles cuja atividade laborativa exige esta postura, são expostos a riscos ocupacionais, sendo mais comum patologias da coluna lombar ${ }^{22}$.

Pesquisadores afirmam que os segmentos da coluna vertebral são suscetíveis a alterações no decorrer da vida devido às adaptações que a vida Ihes impõe. $O$ modelo biomecânico da coluna vertebral não foi feito para permanecer por longos períodos na posição sentada, e explicitam algumas formas de sentar e suas possíveis consequências como fadiga, dores lombares e cãibras ${ }^{23,24}$. A incidência da dor lombar é maior em trabalhadores submetidos a esforços físicos pesados, como levantamento de pesos, movimentos repetitivos e posturas estáticas frequentes ${ }^{25}$.

Apesar das considerações reportadas na literatura a amostra estudada apresentou média de dor lombar na EVA de cinco, o que pode ser considerada dor de média intensidade. Relacionada a esse achado, o resultado para o índice de incapacidade funcional avaliado pelo questionário de Roland Morris e Oswestry também apresentaram escores baixos, 2,8 pontos e $7 \%$, respectivamente.

Este achado pode ser relacionado ao fato das voluntárias constituírem uma população ainda jovem com pouco tempo laboral nesta função. Este achado corrobora com Buscatto26 que aponta que $76 \%$ dos operadores têm menos de 30 anos, $86 \%$ menos de dois anos de experiência em teleatendimento.

A coluna lombar é uma região que faz parte do um complexo lombo-pélvico, descrito na literatura como "centro", uma denominação decorrente do fato de que nesta região fica posicionado o centro de gravidade, onde a maioria dos movimentos é iniciada de acordo com a transmissão de carga do corpo, constituindo assim, uma fonte potencial de dor ${ }^{27}$.

Outra informação identificada nos instrumentos de pesquisa foi o cansaço referido por todas as participantes ao final da carga horária de trabalho sendo, principalmente, o cansaço mental. Os estudos de Torres ${ }^{2}$ e Abrahão et al. ${ }^{28}$ descrevem os sintomas e quadros mórbidos prevalentes, com ênfase para os casos frequentes de sintomas depressivos e sensação de fadiga mental no setor de teleatendimento, com efeitos na esfera da vida extratrabalho. Vilela e Assunção ${ }^{29}$ afirmam em seu estudo que a empresa depende do esforço mental dos teleatendentes para diminuir os efeitos derivados da impossibilidade de garantir a satisfação do cliente nos tempos previstos por suas metas comerciais, causando muita pressão psicológica nos seus funcionários.

Além disso, as centrais de atendimentos esperam que os operadores tenham uma "atitude neutra" em relação ao comportamento dos consumidores, sem deixar transparecer sentimentos desagradáveis ou ruins. Portanto, lidar com todas essas questões exige esforço cognitivo e de controle emocional ${ }^{30}$. O estudo de Corrêa et $\mathrm{al}^{31}$, envolvendo a carga cognitiva da atividade do operador de telemarketing, observou que $53 \%$ da população entrevistada sentem que realizam muito mais do que pode; $63 \%$ sentem-se nervosas por causa do trabalho e $62 \%$ referem-se esgotadas no final do dia.

No Brasil, o Departamento de Saúde e Segurança no Trabalho ligado ao Ministério do Trabalho e Emprego ${ }^{12}$, afirma que a atual forma de organização das centrais de teleatendimento telefônico impõe, de forma simultânea, trabalho sob grande pressão de tempo, elevado esforço mental, elevado esforço visual, exigência de grande responsabilidade acompanhada de falta de controle sobre o processo de trabalho, rigidez postural e sobrecarga estática de segmentos corporais.

Apesar de numerosas causas e fatores de risco que estão relacionados com a lombalgia, Nieman ${ }^{32}$ e Santos $^{33}$ a caracterizam como uma doença de pessoas com vida sedentária; a inatividade física estaria relacionada direta ou indiretamente com dores na coluna; a maior parte da atenção dirige-se a considerá-la um subproduto da combinação da aptidão músculo-esquelética deficiente e uma ocupação que force essa região ${ }^{32,33}$. Num estudo longitudinal realizado por Thorbjornsson ${ }^{34}$, foram pesquisados fatores ocupacionais relacionados com a lombalgia. Os resultados apontaram que, em ambos os sexos, tanto o sedentarismo como o trabalho com grandes cargas, representam indicadores de risco para a lombalgia. Acredita-se que a postura sentada e o sedentarismo podem provocar alterações musculoesqueléticas, como a diminuição dos níveis de força e de amplitude de movimento ${ }^{35}$. 
A principal limitação deste estudo foi o número amostral baixo, porém considerando a relevância do tema para os profissionais da área da saúde, principalmente o fisioterapeuta, devido ao grande número de pacientes com incapacidade devido a dor lombar, sugere-se mais estudos nesta área e com esta população, com um número amostral maior investigando ainda as consequências a longo prazo no desempenho dessa função.

\section{Conclusão}

A dor lombar esteve presente na maioria da amostra estudada, mesmo sendo uma dor de intensidade média; a maioria das voluntárias apresentou um nível de incapacidade baixo. Pode-se observar também que as trabalhadoras desenvolvem suas atividades na posição sentada e possuem pouco tempo de trabalho na empresa e apresentam grande cansaço mental após a jornada de trabalho.

\section{Referências bibliográficas}

1.Michael M. Stress - sinais e causas. Roche; 1998.

2.Torres CC. A atividade nas centrais de atendimento: outra realidade, as mesmas queixas [Dissertação de Mestrado]. Brasília: Instituto de Psicologia,

Universidade de Brasília; 2001.

3.Coury HJC. Perspectivas e Requisitos para a Atuação Preventiva da Fisioterapia nas Lesões Músculo Esqueléticas. Fisioterapia em Movimento. 1992/1993; 5(2):63-8.

4.Rohlmann A. et al. Measured loads on a vertebral body replacement during sitting. The Spine Journal. 2011;11(9):870-5. 5.Fundação Carlos Chagas. Mulheres, trabalho e família. Disponível em: <www.fcc.org.br>.Acesso em: 28/11/2012.

6.Rasch PJ. Cinesiologia e anatomia aplicada. 7 ed . Rio de Janeiro: Guanabara Koogan; 1989.

7.Ladeira CE. Evidence based practice guidelines for management of low back pain: physical therapy implications. Revista Brasileira de Fisioterapia. 2011; 15(3):190-9.

8.Vasconcelos AF. Qualidade de vida no trabalho: origem, evolução e perspectivas. Cadernos de Pesquisa em Administração. 2011;8(1)23-35.

9.Deliberato PCP. Fisioterapia Preventiva. São Paulo: Manole, 2002.

10.Vilela LVO; Assunção AÁ. A atividade do teleatendente: uma interface entre a organização do trabalho e o cliente. Dissertação (Mestrado em Saúde Pública). Faculdade de Medicina da Universidade Federal de Minas Gerais. Belo Horizonte, 2005. 118p.

11.Gallais S. Les Centres d'appels. Provence, Department d'ERGOLOGIE -APST, Université de Provénce;1999.

12.Brasil. Ministério do Trabalho e Emprego. Código Brasileiro de Ocupações, 2002. Disponível em: <http//:www.mtecbo. gov.br>. Acesso em: 01 de junho de 2013.

13.Queiroga MR. Testes e medidas para avaliação da aptidão física relacionada à saúde em adultos. Rio de Janeiro: Guanabara Koogan S. A.; 2005.

14.Pimenta CAM. Escalas de avaliação de dor. In: Teixeira MD (ed.). Dor: conceitos gerais. São Paulo: Limay 1994; 1.46-56. 15.Nusbaum L, Natour J, Ferraz MB, Goldenberg J. Translation, adaptation and validation of the roland-morris questionnaire - Brazil roland-morris. Braz J Med Biol. Res. 2001;34(2):203-10.

16.Roland M, Fairbank J. The Roland-Morris Disability Questionnaire and the Oswestry Disability Questionnaire. Spine. 2003;25(3):3115-3124.

17.Beattie P, MAHER C. The role of functional status questionnaires for low back pain. Aust. Physiother. 1997;43(1):29-38. 18.Rio, Rodrigo Pires; PIRES, Licínica. Ergonomia: fundamentos da prática ergonômica. 2. ed. Belo Horizonte: Health, 1999.

19. Heuch I et al. . The impact of body mass índex on the prevalence of low back pain: the HUNT study. Spine (Phila Pa 1976). 2010;35(7):764-768.

20.Brito, PM, et al. Análise da relação entre a postura de trabalho e a incidência de dores na coluna vertebral. Disponível em: <http://www.abepro.org.br/biblioteca/ENEGEP2003_TR0406_1582.pdf > Data de acesso: 10/12/2012. 
21.Marras W. Biomechanics of the human body. In: Salvendy, Handbook of human factors and Ergonomics , 2 ed. Nova lorque: John Wiley \& Sons; 1997.

22.Viel E.; Esnault M. Lombalgias e cervicalgias da posição sentada: conselhos e exercícios. São Paulo: Editora Manole; 2000.

23.Braccialli LMP.; Vilarta R. Aspectos a serem considerados na elaboração de programas de prevenção e orientação de problemas posturais. Revista Paulista de Educação Física. São Paulo. 2000;14(1):16-28.

24.Knoplich J. Viva bem com a coluna que você tem: dores nas costas, tratamento e prevenção. São Paulo: Ibrasa; 1986. 25.Andrade SC, Araújo AG, VIlar MJ . Escola de Coluna: revisão histórica e sua aplicação na lombalgia crônica. Rev Bras Reumatol. 2005;45(4):224-248.

26.Buscatto M. Les Centres d'appels, usines modernes? Les ationalisations paradoxales de la relation teléphonique. Sociologie du travail. 44 (2002) 99-117. Paris: Éditions Elsévier; 2002.

27.Reinehr FR, Carpes FP, Mota CB. Influência do treinamento de estabilização central sobre a dor e estabilidade lombar. Fisioter Mov. 2008; 21(1):123-9.

28.Abrahão JI, Torres CC, Assunção AA. Penosidade e estratégias de atenuação do risco: o caso das telefonistas de uma instituição pública. Estudos, Vida e Saúde. 2003;v.30:85-110.

29.VIlela LVO, Assunção AA. Queixas de cansaço e esgotamento de trabalhadores do setor de teleatendimento. Cad. Saúde Pública. Rio de Janeiro. 2004; 20(4):1069-1078.

30.Sutton RI. Maintaining norms about expressed emotions: the case of bill collectors. Administrative Science Quarterly.1991; v.36:245-268.

31.Corrêa FP, et al. Avaliação da carga cognitiva em serviços de teleatendimento. In: VI congresso latino-americano de ergonomia (CD-Rom). Gramado: Abergo; 2001.

32.Nieman DC. Exercício e saúde. São Paulo: Manole; 1999.

33.Santos AC. O exercício físico e o controle da dor na coluna vertebral.

Rio de Janeiro: Medsi; 1996.

34.Thorbjornsson CB, et al. Physical and psychosocial factors related to low back pain during a 24-year period. A nested case-control analysis. Spine. 2000; 25:369-74.

35.Reis PF, Moro ARP, Contijo LA. A importância da manutenção de bons níveis de flexibilidade nos trabalhadores que executam suas atividades laborais sentados. Rev Prod On Line. 2003;3(3)1-16.

\section{Hedioneia Maria Foletto Pivetta}

Endereço para correspondência - Rua Recanto Verde, 5, Loteamento Behr. Bairro Camobi, CEP: 97604-105, Santa Maria, RS, Brasil.

E-mail: hedioneia@yahoo.com.br

Lattes: http://lattes.cnpq.br/9518521941876440

Ana Paula Ziegler Vey - aninhaziegler@hotmail.com

Enviado em 02 de abril de 2014.

Publicado em 15 de setembro de 2014. 